\title{
Quadrivalent human papillomavirus vaccination in girls and the risk of autoimmune disorders: the Ontario Grade 8 HPV Vaccine Cohort Study
}

\author{
Erin Y. Liu MSc, Leah M. Smith PhD, Anne K. Ellis MD MSc, Heather Whitaker PhD, Barbara Law MD, \\ Jeffrey C. Kwong MD MSc, Paddy Farrington PhD, Linda E. Lévesque PhD
}

Cite as: CMAJ 2018 May 28;190:E648-55. doi: 10.1503/cmaj.170871

\begin{abstract}
BACKGROUND: Despite demonstrated effectiveness in real-world settings, concerns persist regarding the safety of the quadrivalent human papillomavirus (HPV4) vaccine. We sought to assess the risk of autoimmune disorders following HPV4 vaccination among grade 8 girls eligible for Ontario's school-based HPV vaccination program.
\end{abstract}

METHODS: We undertook a populationbased retrospective cohort study using Ontario's administrative health and vaccination databases from 2007 to 2013. The self-controlled case series method was used to compare the rate of a composite end point of autoimmune disorders diagnosed during days 7-60 post- vaccination ("exposed" follow-up) to that at any other time ("unexposed"). The analysis was repeated to assess the effect of a history of immune-mediated diseases and time since vaccination. We also conducted an exploratory analysis of individual autoimmune disorders. Rate ratios and $95 \%$ confidence intervals (Cls) were estimated using conditional Poisson regression, adjusted for age, seasonality, concomitant vaccinations and infections.

RESULTS: The study cohort consisted of 290939 girls aged $12-17$ years who were eligible for vaccination between 2007 and 2013. There was no significant risk for developing an autoimmune disorder following HPV4 vaccination $(n=681$; rate ratio $1.12,95 \% \mathrm{Cl} 0.85-1.47$ ), and the association was unchanged by a history of immune-mediated disorders and time since vaccination. Exploratory analyses of individual autoimmune disorders found no significant risks, including for Bell palsy ( $n=65$; rate ratio 1.73 , $95 \% \mathrm{Cl} 0.77-3.89$ ), optic neuritis $(n=67$; rate ratio $1.57,95 \% \mathrm{Cl} 0.74-3.33$ ) and Graves disease ( $n=47$; rate ratio 1.55 , 95\% Cl 0.92-2.63).

INTERPRETATION: We did not observe an increased risk of autoimmune disorders following HPV4 vaccination among teenaged girls. These findings should reassure parents and health care providers.
$\mathrm{H}$ uman papillomavirus (HPV) is the most common sexually transmitted infection globally, ${ }^{1}$ affecting $50 \%-75 \%$ of sexually active individuals. ${ }^{2,3}$ The quadrivalent HPV (HPV4) vaccine protects against 2 oncogenic strains of HPV and has been available since 2006. Despite demonstrated effectiveness in real-world settings, ${ }^{4,5}$ including proven reduction in the risk of cervical dysplasia, concerns persist about the vaccine's safety, ${ }^{6,7}$ particularly in light of case reports of autoimmune disorders following HPV vaccination. ${ }^{8-10}$

A recent news story reporting cases of serious debilitating illnesses after HPV4 - although subsequently retracted ${ }^{11}-$ reactivated parental concerns about the safety of this vaccine. ${ }^{12}$ As such, we undertook a population-based, retrospective cohort study to assess the risk of autoimmune disorders following HPV4 vaccination among grade 8 girls eligible for Ontario's HPV vaccination program.

\section{Methods}

\section{Setting}

During the study period (2007-2013), HPV4 vaccination (Gardasil, Merck) was offered free to all grade 8 girls in Ontario through school-based clinics. ${ }^{13}$ This optional vaccine usually required parental consent and was typically administered in September/ October, November/December and March/April, corresponding with the recommended 0-, 2- and 6-month dosing interval for the 
3-dose series used at that time. Eligible girls also had the option of vaccination through their local public health unit or physician at no cost, although most received it at school. During the study, all doses of the HPV4 vaccine were documented in the Immunization Records Information System (IRIS) database regardless of the setting.

\section{Study population and data sources}

We identified a population-based cohort of all girls eligible for Ontario's grade 8 HPV vaccination program between 2007 and 2013 using birth year as a proxy for grade. Since most girls entering grade 8 turn 13 years of age by Dec. 31 of that year, girls eligible for the $2007 / 08$ to $2012 / 13$ vaccination programs were born in 1994 to 1999 . This approach correctly identified an estimated $96.4 \%$ of eligible girls. ${ }^{14}$ We excluded girls who received HPV vaccination before program eligibility and those whose vaccination records were either unavailable (i.e., not yet record-linked with the province's health databases) or inactive (i.e., moved out of Ontario or to a public health unit whose vaccination records were unavailable). Cohort members were followed from Sept. 1 of their grade 8 year (cohort entry) until the earliest of date of death or Mar. 31, 2013 (study end).

The administrative health databases used included the Registered Persons Database, which contains demographic information on all Ontario residents; the Ontario Health Insurance Plan (OHIP) for information on physician services; the National Ambulatory Care Reporting System (NACRS) for information on emergency department visits; and the Discharge Abstract Database (DAD) for detailed information on hospital admissions. These databases were accessed through the Institute for Clinical Evaluative Sciences (ICES).

Each public health unit maintains its own IRIS database to record information on vaccines administered through the province's publicly funded program. At the time of this study, a copy of IRIS from 32 of Ontario's 36 public health units had been transferred to ICES, where records were linked and anonymized. A validation study performed using standard methods and the paper vaccination records as the gold standard found that doses of the HPV4 vaccine in IRIS were captured with high sensitivity (99.8\%, 95\% confidence interval [Cl] 99.34\%-99.95\%) and high specificity $(95.4 \%, 95 \% \mathrm{Cl} 93.7 \%-96.85)$, and that $98.6 \%$ of vaccination dates were accurate. ${ }^{15}$

\section{Study outcomes}

The primary outcome was a first diagnosis of any of 12 autoimmune disorders included in the composite end point (Appendix 1, available at www.cmaj.ca/lookup/suppl/doi:10.1503/ cmaj.170871/-/DC1). The included autoimmune disorders comprised those commonly targeted for postmarketing surveillance, ${ }^{16,17}$ identified in case reports for the HPV4 vaccine, ${ }^{8,9,18-23}$ or reported to the US Vaccine Adverse Event Reporting System (VAERS) database. ${ }^{24,25}$ A composite end point was chosen because most autoimmune disorders are rare. This approach also reduced the potential for misclassification since some autoimmune disorders present with similar symptoms, lack definitive diagnostic tests and may take time to diagnose correctly.
Autoimmune disorders were identified using diagnostic codes in the OHIP, NACRS and DAD databases determined in consultation with clinical experts (Appendix 2, available at www.cmaj.ca/ lookup/suppl/doi:10.1503/cmaj.170871/-/DC1). To capture only incident cases, we excluded girls with a diagnosis of any autoimmune disorder before cohort entry. Since few of the diagnostic codes used have been validated, a validated algorithm for identifying systemic autoimmune rheumatic diseases using administrative health data was adapted for this study (Appendix 2). ${ }^{26}$ This algorithm has a sensitivity of $83 \%$ and a specificity ranging from $72.5 \%$ to $96.4 \%$ for individual systemic autoimmune rheumatic diseases. $^{26}$

\section{Statistical analysis}

Studies on vaccine safety typically compare cohorts of vaccinated and unvaccinated populations. However, such comparisons are particularly prone to confounding bias ${ }^{27}$ when the risk factors for the outcome of interest are largely unknown or difficult to quantify (e.g., genetic susceptibility), as with autoimmune disorders. To circumvent this bias, we used the selfcontrolled case series method developed specifically for vaccine safety studies. ${ }^{28}$ With this method, vaccinated cases serve as their own controls because their follow-up time can be divided into "exposed" and "unexposed" time intervals. The exposed time intervals (also known as "exposure risk windows") correspond to periods postvaccination that are biologically attributable to the effects of the vaccine, and unexposed follow-up represents time intervals preceding vaccination and following the exposure risk window (Appendix 3, available at www.cmaj.ca/lookup/suppl/doi:10.1503/cmaj.170871/-/DC1). As such, vaccinated cases are compared with themselves over time and unvaccinated cases are not required. The incidence rate during exposed follow-up time is compared with that during unexposed follow-up, and rate ratios and $95 \% \mathrm{Cls}$ are estimated using conditional Poisson regression to account for the self-matching.

The 3 key assumptions of the self-controlled case series method are (1) outcomes are either recurrent and independent, or unique and rare; (2) outcomes must not censor follow-up (i.e., cannot be fatal); and (3) outcomes must not affect the probability of future vaccination. In our study, assumptions 1 and 2 were fulfilled given that autoimmune disorders are rare and usually nonfatal, and assumption 3 was also met since most girls received all 3 doses of the HPV4 vaccine and as many outcomes were diagnosed after the third dose as with the first and second doses.

The exposure risk window for the primary analysis was 7-60 days following each dose. This risk window was based on the underlying pathophysiology and clinical manifestation of the autoimmune disorders studied, including their insidious onset; ${ }^{29}$ the risk window used in studies of autoimmune disorders following use of other vaccines (Appendix 4, available at www. cmaj.ca/lookup/suppl/doi:10.1503/cmaj.170871/-/DC1); data on time-to-onset of autoimmune diseases following HPV4 vaccination reported to the VAERS database; ${ }^{24}$ and recognition that health databases capture time to medical contact rather 
than time to onset of symptoms. All other follow-up time was considered unexposed.

The self-matched nature of the self-controlled case series method implicitly controlled for all time-fixed confounders, including ethnicity, genetic susceptibility, personal beliefs and values, and health behaviours. However, we controlled for the potentially confounding effects of time-varying risk factors, including age at diagnosis ( $\leq 14,15-16, \geq 17 \mathrm{yr}$ ), seasonality as a proxy for influenza season and vaccination (September to December as individual months, January-February and MarchAugust combined), recent infections (7-60 d before diagnosis), and receipt of concomitant vaccines (7-60 d before diagnosis) by including these factors in the Poisson models.

To identify subgroups at potentially higher risk for autoimmune disorders following HPV4 vaccination, we repeated the primary analysis stratified by a history of immune-mediated diseases (i.e., asthma, anaphylaxis and other atopic manifestations). Also, to determine whether the risk for autoimmune disorders postvaccination varied over time, the primary exposure risk window was stratified into the following periods: 7-24, 25-42 and 43-60 days. Finally, the analysis was repeated for individual autoimmune disorders.

We tested the robustness of our results through planned sensitivity analyses. First, to determine whether any etiologically relevant outcomes were excluded from the primary end point, the analysis was repeated using an expanded composite end point (Appendix 1). Second, to address the concern that disease onset may have preceded HPV4 vaccination, the analysis was repeated excluding cases diagnosed within 30 days after vaccination. Finally, to address the possibility of exposure misclassification due to an erroneously specified exposure risk window, the 7-60 days risk window was widened to 0-60, 7-90 and $7-180$ days. These risk windows have been used in previous vaccine studies of autoimmune disorders (Appendix 4).

This study had more than $90 \%$ power to detect a rate ratio of 2.0 for autoimmune disorders. Statistical analyses were conducted using SAS version 9.3 and Stata version 11.

\section{Ethics approval}

The Queen's University Health Sciences and Affiliated Teaching Hospitals Research Ethics Board approved this study.

\section{Results}

The cohort consisted of 290939 girls eligible for Ontario's grade 8 HPV vaccination program between 2007 and 2013 (Figure 1). The mean age was 13.2 (range 12.7-13.7) years at cohort entry, and the girls were followed for a mean of 2.9 (standard deviation 1.5) years. A total of 180819 (62.2\%) girls received at least 1 dose of the HPV4 vaccine, with most receiving all 3 doses $(81.8 \%)$, and $110120(37.8 \%)$ were unvaccinated. Although the proportion of unvaccinated girls may seem high, it reflects the welldocumented low uptake of this vaccine during the initial years of the program. ${ }^{13,30}$

Among 681 incident cases of autoimmune disorders diagnosed in vaccinated girls, $77(11.3 \%)$ occurred $7-60$ days after HPV4 vaccination (i.e., exposed cases) (Figure 2). Exposed cases were as likely to be diagnosed after the first dose $(n=21)$ as the second $(n=26)$ or third dose $(n=30)$, and most $(79.2 \%)$ exposed cases received all 3 doses. The end point consisted primarily of cases of juvenile rheumatoid arthritis, followed by systemic autoimmune rheumatic diseases, immune thrombocytopenic purpura, optic neuritis and Bell palsy (Table 1); the pattern is consistent with the background incidence of these disorders in this age group. ${ }^{31}$

Age at diagnosis, history of recent infections and history of immune-mediated disease were significant risk factors for autoimmune disorders, whereas vaccination with a non-HPV vaccine was not (Table 2). With the exception of October, seasonality was not significantly associated with the risk of autoimmune disorders. Nevertheless, all of these factors were included in the analysis.

Receipt of the HPV4 vaccine was not associated with an increased risk of developing an autoimmune disorder (adjusted rate ratio $1.12,95 \% \mathrm{Cl} 0.85-1.47$ ), and the association was independent of a girl's history of immune-mediated diseases ( $p=$ 0.4) (Table 3).

When stratified by time since vaccination, the adjusted rate ratios increased from 0.95 to 1.31 , then decreased to 0.97 for the risk windows of 7-24, 25-42 and 43-60 days, respectively; however, none of these differences were significant (Table 4).

The exploratory analysis of individual autoimmune disorders found no significantly increased risks, including for Bell palsy, optic neuritis and Graves disease (Figure 3).

The sensitivity analyses using the expanded outcome definition and excluding cases occurring within 30 days of the first HPV4 dose were essentially unchanged (adjusted rate ratios 1.05, 95\% Cl 0.72-1.55; and 0.97, 95\% Cl 0.73-1.30, respectively). The association between HPV4 vaccination and autoimmune disorder was also unaffected by the inclusion of the 7 days immediately following HPV4 vaccination (adjusted rate ratio $0.99,95 \% \mathrm{Cl} 0.76-1.30$ ), and the addition of 30 days and 120 days to the primary exposure risk window (adjusted rate ratios 0.98 , 95\% $\mathrm{Cl} 0.76-1.26$; and $0.94,95 \% \mathrm{Cl} 0.75-1.17$, respectively).

\section{Interpretation}

We did not find a significantly increased risk for autoimmune disorders following HPV4 vaccination in this large population of girls aged 12-17 years, including in those with pre-existing immune-mediated diseases. The association was also unaffected by time since vaccination. Moreover, our results were robust to numerous sensitivity analyses.

Our results are similar to those of 2 other large cohort studies of the HPV4 vaccine carried out using the population-based health care registries of Denmark and Sweden. The first study followed 997585 girls aged 10-17 years and did not find a significant risk for the autoimmune disorders assessed, ${ }^{32}$ and the second followed 3.9 million girls and women 10-44 years of age and reported no increased risk for multiple sclerosis and other demyelinating diseases of the central nervous system. ${ }^{33}$ In addition, 2 small case-control studies involving older age 


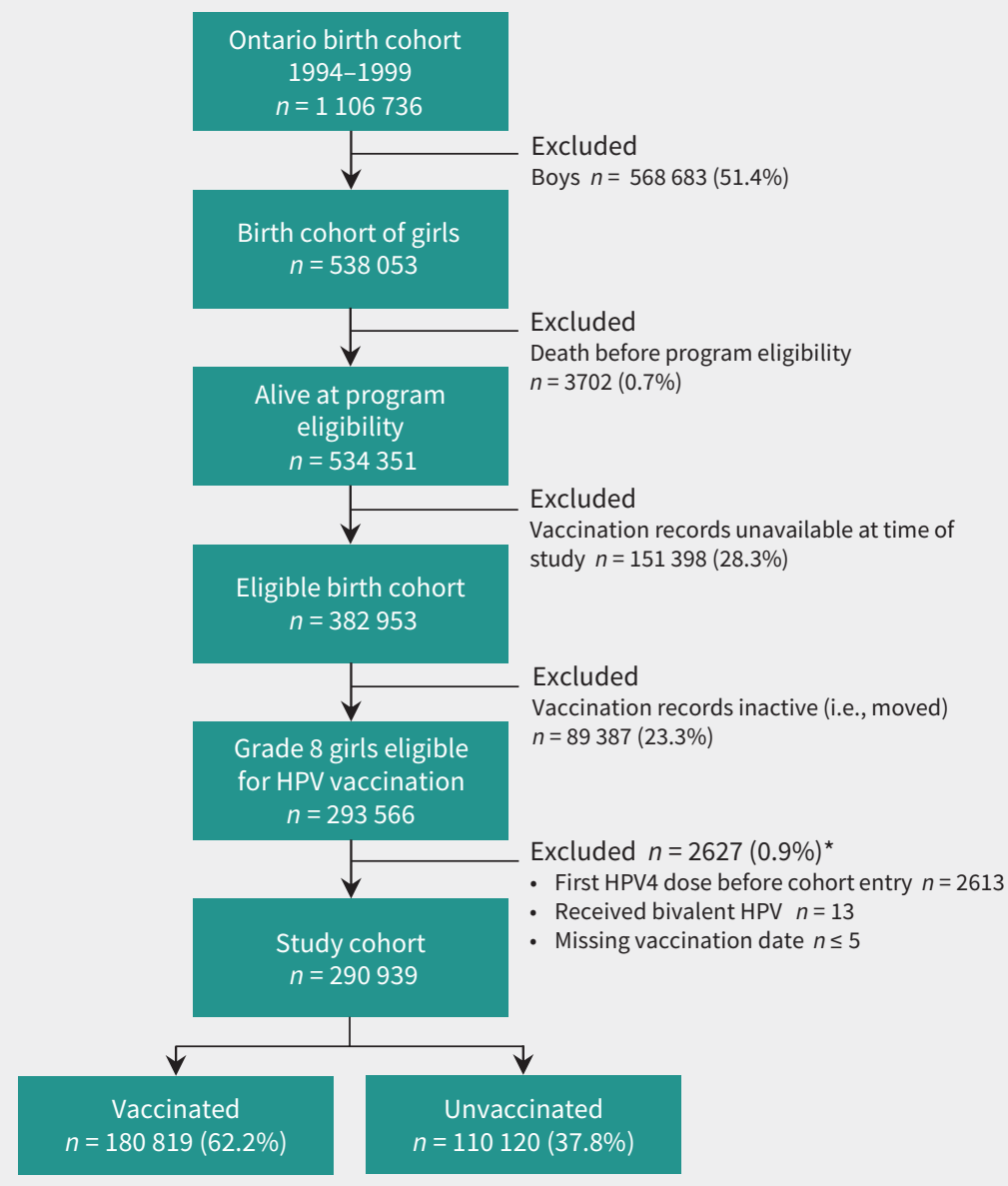

Figure 1: Cohort flow diagram. ${ }^{*}$ Cohort members could fulfill more than 1 exclusion criteria; therefore, criteria were not mutually exclusive. Note: HPV = human papillomavirus, HPV4 = quadrivalent HPV.

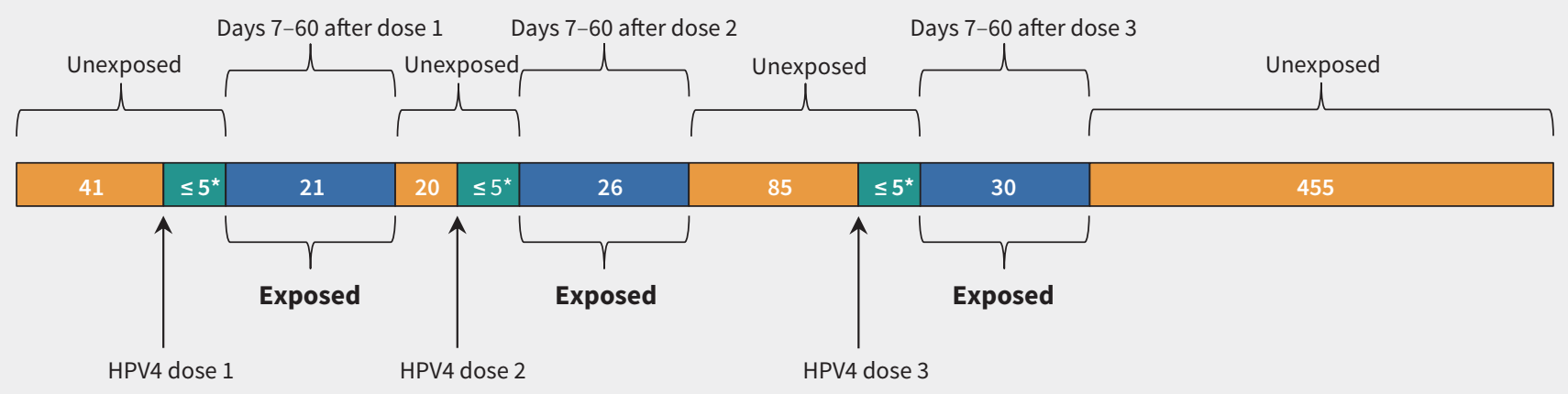

Unexposed person-time, where the occurrence of an event would not be attributable to the HPV4 vaccine

Unexposed person-time (0-6 d postvaccination), to account for the time it takes for symptoms to develop

Exposed person-time (7-60 d postvaccination), where events occurring during this period are attributable to the effects of HPV4 vaccine

Figure 2: Distribution of exposed and unexposed cases of autoimmune disorders after each dose of the quadrivalent human papillomavirus (HPV4) vaccine. ${ }^{*}$ Counts of fewer than 5 cases were suppressed owing to data privacy guidelines. 
groups, conducted in France ${ }^{34}$ and the United States, ${ }^{35}$ also reported no increased risk for various autoimmune disorders. However, the studies published to date have been limited by

Table 1: Incident cases of autoimmune disorders included in the composite end point

\begin{tabular}{|c|c|}
\hline Autoimmune disorder & $\begin{array}{l}\text { No. }(\%) \text { of vaccinated cases } \\
\qquad n=681^{\star}\end{array}$ \\
\hline Juvenile rheumatoid arthritis & $323(44.6)$ \\
\hline Immune thrombocytopenia purpura & $103(15.1)$ \\
\hline Bell palsy & $65(9.0)$ \\
\hline $\begin{array}{l}\text { Systemic autoimmune rheumatic } \\
\text { diseases } \dagger\end{array}$ & $111(15.4)$ \\
\hline Multiple sclerosis & $17(2.4)$ \\
\hline Optic neuritis & $67(9.3)$ \\
\hline $\begin{array}{l}\text { Acute disseminated } \\
\text { encephalomyelitis }\end{array}$ & $21(2.9)$ \\
\hline Autoimmune hepatitis & $12(1.7)$ \\
\hline Guillain-Barré syndrome & $\leq 5(\leq 0.7)$ \\
\hline Neuromyelitis optica & 0 \\
\hline Transverse myelitis & $\leq 5(\leq 0.7)$ \\
\hline Autoimmune pancreatitis & 0 \\
\hline \multicolumn{2}{|c|}{$\begin{array}{l}\text { *Proportions are among all vaccinated cases. Column total for vaccinated cases is } \\
\text { greater than } 681 \text { because some girls were diagnosed with } 2 \text { or more autoimmune } \\
\text { disorders during follow-up. } \\
\text { †Includes systemic lupus erythematosus, systemic sclerosis, Sjögren syndrome, } \\
\text { dermatomyositis and polymyositis. }\end{array}$} \\
\hline
\end{tabular}

their use of longer-than-recommended risk windows ${ }^{32-34}$ that could bias toward the null, a lack of validation of vaccination records ${ }^{32,33}$ and a direct comparison of vaccinated and unvaccinated girls, ${ }^{32-34}$ an approach prone to bias. Our study using more robust methods nevertheless found similar results. As such, the consistency of results across different study designs, analytical strategies, risk windows and populations is reassuring.

Recently, a cohort study conducted in France involving 2.2 million girls aged 13-16 years reported no increased risk for 12 of 14 autoimmune disorders evaluated following HPV vaccine composed of either bivalent HPV (HPV2) or HPV4. ${ }^{36}$ Although this study reported a significantly higher risk of Guillain-Barré syndrome among HPV-vaccinated girls compared with unvaccinated girls (adjusted hazard ratio 3.78, $95 \% \mathrm{Cl}$ 1.79-7.98), a UK study of HPV2 and HPV4 vaccination using the more robust self-controlled case series method failed to find such an association (rate ratio $1.04,95 \% \mathrm{Cl} 0.47-2.28$ ). ${ }^{37}$ Although these studies are not directly comparable to ours given their inclusion of the HPV2 vaccine, they nevertheless provide additional evidence against HPV vaccine-induced autoimmune disorders.

\section{Limitations}

Our study benefits from the use of population-based databases, which limits the potential for selection bias, and from the use of a robust analytical method that implicitly controls for all known and unknown time-fixed confounders. Nevertheless, our study had some limitations. First, although the outcome definition was based on an algorithm that has been validated for certain

\section{Table 2: Determinants of autoimmune disorders}

Rate ratio $(95 \% \mathrm{Cl})$

Variable

Age at outcome development, yr

$\leq 14$ (reference)

$15-16$

$\geq 17$

\section{Seasonality}

September (reference)

October

November

December

January to February

March to August

Receipt of other vaccines $\dagger$

History of recent infectionsł

History of immune-mediated disease§
1.00

$1.53(1.21-1.93)$

$2.02(1.53-2.66)$

1.00

Univariate model

Fully adjusted model

$0.75(0.54-1.05)$

$1.25(0.92-1.70)$

$1.02(0.75-1.38)$

$1.04(0.79-1.35)$

$1.22(0.97-1.53)$

$0.87(0.55-1.38)$

$1.49(0.85-2.60)$

$1.50(1.05-2.14)$
1.00

$1.52(1.20-1.92)$

$1.98(1.50-2.62)$

1.00

$0.65(0.45-0.96)$

$1.17(0.84-1.62)$

$0.85(0.60-1.22)$

$0.83(0.61-1.12)$

$0.96(0.75-1.25)$

$2.15(1.33-3.47)$

$1.56(1.11-2.21)$
$0.84(0.53-1.33)$

Note: $\mathrm{Cl}=$ confidence interval, $\mathrm{HPV}=$ human papillomavirus.

${ }^{\star}$ Adjusted for all the factors listed in the table.

†Includes any non-HPV vaccine, such as diphtheria, pertussis, tetanus; measles-mumps-rubella; hepatitis B; and meningococcal C vaccines.

łIncludes infections previously shown to be associated with autoimmune disorders, including cytomegalovirus, parvovirus B19, hepatitis B and C viruses, coxsackievirus B, influenza type A, Campylobacter jejuni, Staphylococcus aureus and Haemophilus influenzae.

§Includes a diagnosis for asthma, anaphylaxis, urticaria, angioneurotic edema, eczema, dermatitis, rash, allergic rhinitis or hay fever before cohort entry. 
Table 3: Autoimmune disorders diagnosed after quadrivalent human papillomavirus vaccination

\section{Cases/person-time}

\begin{tabular}{|c|c|c|c|c|}
\hline Variable & Exposed & Unexposed & Unadjusted* & Adjusted $\dagger$ \\
\hline HPV4 vaccine (overall) & $77 / 99841$ & $604 / 825160$ & $0.86(0.67-1.10)$ & $1.12(0.85-1.47)$ \\
\hline \multicolumn{5}{|c|}{ History of immune-mediated diseases } \\
\hline Yes & $41 / 54247$ & $326 / 446732$ & $0.84(0.61-1.18) \ddagger$ & $1.11(0.72-1.62) \ddagger$ \\
\hline No & $36 / 45567$ & $278 / 378428$ & $0.87(0.61-1.24) \ddagger$ & $1.12(0.75-1.67) \ddagger$ \\
\hline
\end{tabular}

Rate ratio $(95 \% \mathrm{Cl})$

Note: $\mathrm{Cl}$ = confidence interval, $\mathrm{HPV}=$ human papillomavirus, HPV4 = quadrivalent $\mathrm{HPV}$.

*Unadjusted rate ratios were derived from self-matched models that implicitly controlled for time-fixed confounders and only included the HPV vaccine (exposure).

†Adjusted for age at diagnosis, seasonality (Table 2), receipt of non-HPV vaccines (7-60 d before diagnosis) and recent infection (7-60 d before diagnosis), and implicitly adjusted for time-fixed confounders through the use of a self-matched analysis.

$\ddagger p=0.4$ for a 2-sided test of interaction comparing those with and without a history of immune-mediated diseases at a significance threshold of $\alpha=0.05$.

Table 4: Autoimmune disorders diagnosed after quadrivalent human papillomavirus vaccination, stratified by time since vaccination

\section{Rate ratio $(95 \% \mathrm{CI})$}

\begin{tabular}{|c|c|c|c|c|}
\hline Exposure risk window, $\mathrm{d}^{\star}$ & No. of exposed cases & Unadjusted $†$ & Adjusted $\ddagger$ & $p$ value§ \\
\hline $7-24$ & 22 & $0.87(0.49-1.11)$ & $0.95(0.61-1.48)$ & \multirow{3}{*}{$\begin{array}{l}0.1 \\
0.2\end{array}$} \\
\hline $25-42$ & 40 & $1.38(0.74-1.42)$ & $1.31(0.93-1.84)$ & \\
\hline $43-60$ & 15 & $0.73(0.44-1.42)$ & $0.97(0.57-1.66)$ & \\
\hline
\end{tabular}

Note: $\mathrm{Cl}=$ confidence interval, $\mathrm{HPV}=$ human papillomavirus, $\mathrm{HPV} 4$ = quadrivalent $\mathrm{HPV}$.

*The duration of time following each dose of the HPV4 vaccine during which the occurrence of an event is attributable to the effects of the vaccine.

†Unadjusted rate ratios were derived from self-matched models that implicitly controlled for time-fixed confounders and only included the HPV vaccine (exposure). łAdjusted for age at diagnosis, seasonality (Table 2), receipt of non-HPV vaccines (7-60 d before diagnosis) and recent infection (7-60 d before diagnosis), and implicitly adjusted for time-fixed confounders through the use of a self-matched analysis.

$\S p$ value for 2 -sided test of interaction between risk windows using a significance threshold of $\alpha=0.05$.

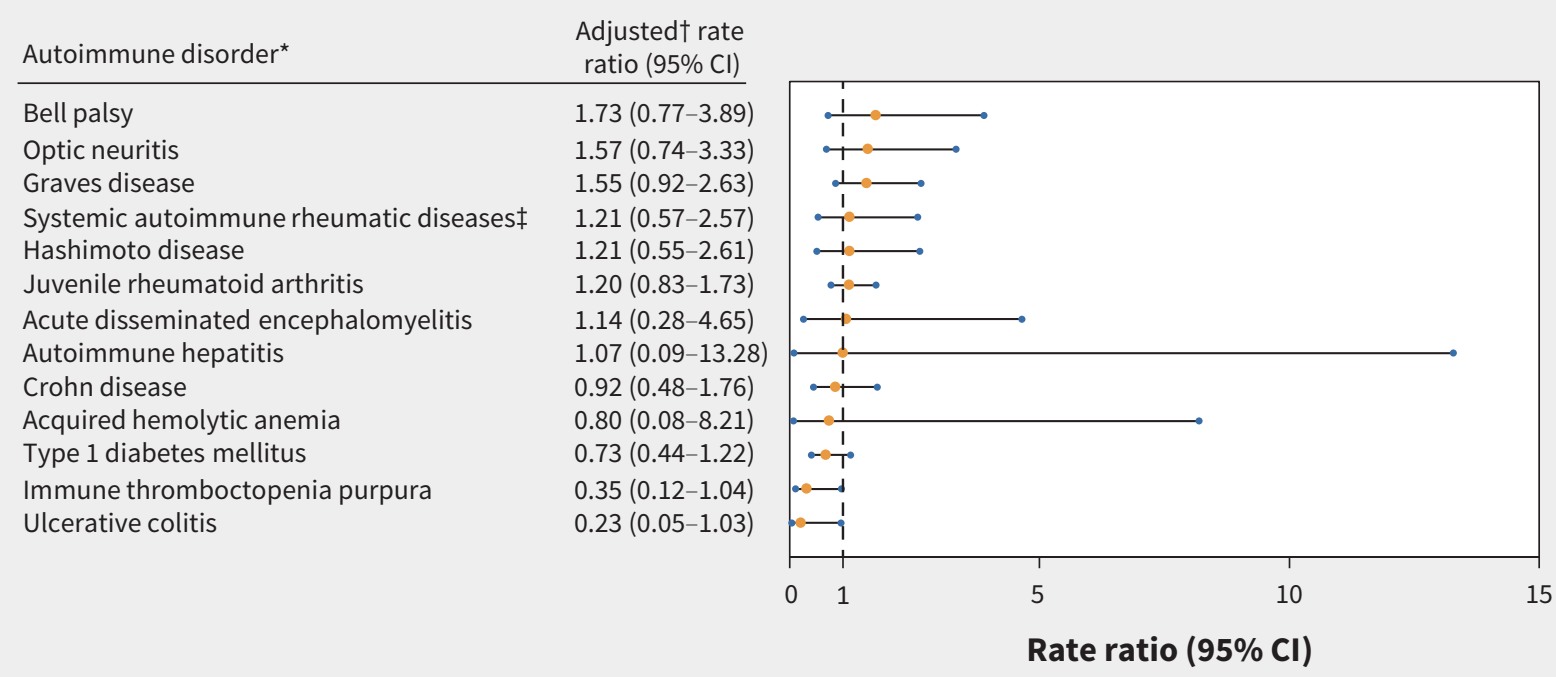

Figure 3: Adjusted rate ratios for individual autoimmune disorders diagnosed in the 7-60 days after quadrivalent human papillomavirus (HPV4) vaccination. *Autoimmune disorders with 10 or more vaccinated cases. †Adjusted for age at diagnosis, seasonality (Table 2), receipt of non-HPV vaccines (7-60 d before diagnosis) and recent infection (7-60 d before diagnosis), and implicitly adjusted for time-fixed confounders through the use of a self-matched analysis. łIncludes systemic lupus erythematosus, systemic sclerosis, Sjögren syndrome, dermatomyositis and polymyositis. Note: $\mathrm{Cl}=$ confidence interval. 
autoimmune disorders, the available data did not allow us to validate the disorders included in the composite end point. We expect any misclassification to be nondifferential (i.e., unrelated to timing of vaccination) and, therefore, to bias toward the null. Second, the date of symptom onset was not available. To address the concern that disease onset may have preceded HPV4 vaccination, we repeated the analysis excluding diagnoses occurring within 30 days of the first dose and found similar results. Third, the rarity of the outcomes necessitated the use of a composite end point and, although not ideal, the exploratory analysis of individual disorders supported our primary findings. Fourth, there is potential for exposure misclassification given the use of the same risk window for all autoimmune disorders included in the composite end point. However, our results were robust despite the use of varying time windows. Fifth, although our use of a self-matched analysis implicitly controls for timefixed confounders, and the analysis included known timevarying factors, we cannot rule out the possibility of confounding from unknown time-varying factors. As such factors are unlikely, the potential for residual confounding is low. Finally, our results may not be generalizable to other populations, including older women and boys, for whom the HPV vaccine is also indicated. However, evidence does not suggest they are at higher risk for vaccine-induced autoimmune disorders. Also, although only half of girls eligible for Ontario's program were included in this study owing to data availability, the girls included were from geographically, ethnically and economically diverse public health units of varying sizes. As such, our cohort is likely representative of all eligible girls.

\section{Conclusion}

This large, population-based study did not find a significant risk of autoimmune disorders following HPV4 vaccination among girls aged 12-17 years, including girls with a history of immune-mediated disorders. These findings add to the growing body of evidence on the safety of this vaccine and should reassure parents and health care providers.

\section{References}

1. Trottier H, Franco E. The epidemiology of genital human papillomavirus infection. Vaccine 2006;24(Suppl 1):S1-15.

2. Baseman JG, Koutsky L. The epidemiology of human papillomavirus infections. J Clin Virol 2005;32:(Suppl 1):S16-24.

3. Tota JE, Chevarie-Davis M, Richardson L, et al. Epidemiology and burden of HPV infection and related diseases: implications for prevention strategies. Prev Med 2011;53:(Suppl 1):S12-21.

4. Smith LM, Strumpf EC, Kaufman JS, et al. The early benefits of human papillomavirus vaccination on cervical dysplasia and anogenital warts. Pediatrics 2015;135:e1131-40 10.1542/peds.2014-2961.

5. Smith LM, Kaufman JS, Strumpf EC, et al. Effect of human papillomavirus (HPV) vaccination on clinical indicators of sexual behaviour among adolescent girls: the Ontario Grade 8 HPV Vaccine Cohort Study. CMAJ 2015;187:E74-81.

6. Trim K, Nagji N, Elit L, et al. Parental knowledge, attitudes, and behaviours towards human papillomavirus vaccination for their children: a systematic review from 2001 to 2011. Obstet Gynecol Int 2012;2012:921236.

7. Feinberg Y, Pereira JA, Quach S, et al. Understanding public perceptions of the HPV vaccination based on online comments to Canadian news articles. PLOS One 2015;10:e0129587.
8. Soldevilla HF, Briones S, Navarra S. Systemic lupus erythematosus following HPV immunization or infection? Lupus 2012;21:158-61.

9. Sutton I, Lahoria R, Tan I, et al. CNS demyelination and quadrivalent HPV vaccination. Mult Scler 2009;15:116-9.

10. Naleway AL, Gold R, Drew L, et al. Reported adverse events in young women following quadrivalent human papillomavirus vaccination. J Womens Health (Larchmt) 2012;21:425-32.

11. Mclean J, Bruser D. A wonder drug's dark side. The Toronto Star 2015 Feb. 5.

12. Hoffman K. The Gardasil girls: How Toronto Star story on young women hurt public trust in vaccine. CBC News 2015 Feb. 14. Available: www.cbc.ca/news/ canada/manitoba/the-gardasil-girls-how-toronto-star-story-on-young-women -hurt-public-trust-in-vaccine-1.2957524. (accessed 2017 June 15).

13. Wilson SE, Harris T, Sethi P, et al. Coverage from Ontario, Canada's schoolbased HPV vaccine program: the first three years. Vaccine 2013;31:757-62.

14. Lévesque LE, Smith LM, Perry AG, et al. The Ontario grade 8 HPV vaccine cohort study: a feasibility and validity evaluation. Toronto: Ontario Vaccine Sciences Symposium; 2011.

15. Smith LM, Lévesque LE, Nasr M, et al. Validity of the Immunization Record Information System (IRIS) database for epidemiologic studies of the human papillomavirus (HPV) vaccine. Can J Clin Pharmacol 2010;171:e90-127.

16. Siegrist C-A, Lewis E, Eskola J, et al. Human papilloma virus immunization in adolescent and young adults: a cohort study to illustrate what events might be mistaken for adverse reactions. Pediatr Infect Dis J 2007;26:979-84.

17. Chao C, Klein N, Velicer C, et al. Surveillance of autoimmune conditions following routine use of quadrivalent human papillomavirus vaccine. J Intern Med 2012;271:193-203.

18. Della Corte C, Carlucci A, Francalanci P, et al. Autoimmune hepatitis type 2 following anti-papillomavirus vaccination in a 11-year-old girl. Vaccine 2011;29:4654-6.

19. Wildemann B, Jarius S, Hartmann M, et al. Acute disseminated encephalomyelitis following vaccination against human papilloma virus. Neurology 2009;72:2132-3.

20. Das A, Chang D, Biankin A, et al. Pancreatitis following human papillomavirus vaccination. Med J Aust 2008;189:178.

21. Chang J, Campagnolo D, Vollmer T, et al. Demyelinating disease and polyvalent human papilloma virus vaccination. J Neurol Neurosurg Psychiatry 2011;82:1296-8

22. Longueville C, Doffoel-Hantz V, Hantz S, et al. Gardasil ${ }^{\circledR}$-induced erythema nodosum [article in French]. Rev Med Interne 2012;33:e17-8.

23. Pugnet G, Ysebaert L, Bagheri H, et al. Immune thrombocytopenic purpura following human papillomavirus vaccination. Vaccine 2009;27:3690.

24. Vaccine Adverse Event Reporting System. Atlanta: Centers for Disease Control and Prevention; Silver Spring (MD): US Food and Drug Administration.

25. Slade BA, Leidel L, Vellozzi C, et al. Postlicensure safety surveillance for quadrivalent human papillomavirus recombinant vaccine. JAMA 2009;302: $750-7$

26. Bernatsky S, Linehan T, Hanly J. The accuracy of administrative data diagnoses of systemic autoimmune rheumatic diseases. J Rheumatol 2011;38:1612-6.

27. Fine PE, Chen R. Confounding in studies of adverse reactions to vaccines. Am J Epidemiol 1992;136:121-35.

28. Farrington $\mathrm{CP}$. Relative incidence estimation from case series for vaccine safety evaluation. Biometrics 1995;51:228-35.

29. Sejvar JJ, Kohl KS, Gidudu J, et al. Guillain-Barré syndrome and Fisher syndrome: case definitions and guidelines for collection, analysis, and presentation of immunization safety data. Vaccine 2011;29:599-612.

30. Remes O, Smith LM, Alvarado-Llano BE, et al. Individual- and regional-level determinants of human papillomavirus (HPV) vaccine refusal: the Ontario Grade 8 HPV vaccine cohort study. BMC Public Health 2014;14:1047.

31. Klein NP, Ray P, Carpenter D, et al. Rates of autoimmune diseases in Kaiser Permanente for use in vaccine adverse event safety studies. Vaccine 2010 28:1062-8. 
32. Arnheim-Dahlström L, Pasternak B, Svanström H, et al. Autoimmune, neurological, and venous thromboembolic adverse events after immunisation of adolescent girls with quadrivalent human papillomavirus vaccine in Denmark and Sweden: cohort study. BMJ 2013;347:f5906.

33. Scheller NM, Svanström H, Pasternak B, et al. Quadrivalent HPV vaccination and risk of multiple sclerosis and other demyelinating diseases of the central nervous system. JAMA 2015;313:54.

34. Grimaldi-Bensouda L, Guillemot D, Godeau B, et al. Autoimmune disorders and quadrivalent human papillomavirus vaccination of young female subjects. J Intern Med 2014;275:398-408.
35. Langer-Gould A, Qian L, Tartof SY, et al. Vaccines and the risk of multiple sclerosis and other central nervous system demyelinating diseases. JAMA Neurol 2014;71:1506.

36. Miranda S, Chaignot C, Collin C, et al. Human papillomavirus vaccination and risk of autoimmune diseases: a large cohort study of over 2 million young girls in France. Vaccine 2017;35:4761-8.

37. Andrews N, Stowe J, Miller E. No increased risk of Guillain-Barré syndrome after human papilloma virus vaccine: a self-controlled case-series study in England. Vaccine 2017;35:1729-32.
Competing interests: Leah Smith is now employed by the Canadian Cancer Society, a charitable organization that promotes HPV vaccination and has received funds from Merck. However, this study was completed before Dr. Smith's employment at the Canadian Cancer Society. Anne Ellis reports funding from ALK-Abelló, AstraZeneca, Bayer, Merck, Novartis, Pediapharm, Pfizer, Sanofi, Takeda and SunPharma. Linda Lévesque is now employed by Sanofi Pasteur, a global vaccine manufacturer; however, this study was completed before her employment at Sanofi Pasteur. No other competing interests were declared.

This article has been peer reviewed.

Affiliations: Department of Public Health Sciences (Liu, Lévesque), Queen's University, Kingston, Ont.; Department of Epidemiology, Biostatistics and Occupational Health (Smith), McGill University, Montréal, Que. Division of Allergy and Immunology, Department of Medicine (Ellis), Queen's University, Kingston, Ont.; Allergy Research Unit (Ellis), Kingston General Hospital, Kingston, Ont.; School of Mathematics and Statistics (Whitaker, Farrington), The Open University, Milton Keynes, UK; Vaccine Safety Section, Centre for Immunization and Respiratory Infectious Diseases (Law [retired from the Public Health Agency of Canada June 2015]), Public Health Agency of Canada, Ottawa, Ont.; Institute for Clinical Evaluative Sciences (Kwong, Lévesque), Toronto, Ont.; Public Health Ontario
(Kwong), Toronto, Ont.; Department of Family and Community Medicine (Kwong), University of Toronto, Toronto, Ont.; Leslie Dan Faculty of Pharmacy (Lévesque), University of Toronto, Toronto, Ont.

Contributors: Erin Liu, Leah Smith, Heather Whitaker, Jeffrey Kwong, Paddy Farrington and Linda Lévesque contributed substantially to the conception and design of the study. Leah Smith and Linda Lévesque contributed to the acquisition of data. Erin Liu, Leah Smith, Anne Ellis, Heather Whitaker, Barbara Law, Paddy Farrington and Linda Lévesque contributed to the analysis and interpretation of data. Erin Liu drafted the article, which all of the authors revised critically for important intellectual content. All of the authors gave final approval of the version to be published and agreed to act as guarantors of the work.

Funding: This study was funded by grants from the Ontario Ministry of Health and Longterm Care, Drug Innovation Fund (MOHLTCDIF \#2009-003) and the Canadian Institutes of Health Research (CIHR \#MOP-106553). The sponsors had no role in the design, conduct, analysis, interpretation, preparation, review or approval of the manuscript, or the decision to submit the manuscript for publication.

Acknowledgements: Erin Liu was supported by the CIHR Frederick Banting and Charles Best Master's Award. Leah Smith was supported by the CIHR Frederick Banting and Charles Best Doctoral Award. Linda Lévesque is supported by the Canadian Foundation for Innovation (CFI) Leaders Opportunity Fund and the Ontario Ministry of Research, Innovation and Science Ontario Research Fund for research infrastructure. Anne Ellis is also supported by infrastructure grants from the CFI and the Ontario Ministry of Research, Innovation and Science, as well as The Allergy, Genes and Environment Network of Centers of Excellence and the Physicians' Services Incorporated Foundation. Jeffrey Kwong is supported by a New Investigator Salary Award from CIHR and a Clinician Scientist Salary Award from the Department of Family and Community Medicine, University of Toronto.

Disclaimer: This study was supported by the Institute for Clinical Evaluative Sciences (ICES), which is funded by an annual grant from the Ontario Ministry of Health and Long-term Care (MOHLTC). Parts of this material are based on data and information compiled and provided by the Canadian Institute for Health Information (CIHI). The opinions, results and conclusions reported in this paper are those of the authors and are independent from the funding sources. No endorsement by ICES, $\mathrm{CIHI}$ or the Ontario MOHLTC is intended or should be inferred.

Accepted: Feb. 27, 2018

Correspondence to: Linda Lévesque, linda.levesque@utoronto.ca 